\title{
A Single 10k Run Session does not Change either Skinfold Thickness or Body Circumferences
}

\author{
João Gabriel da Silveira-Rodrigues ${ }^{1, *}$, Camila Cristina Melo ${ }^{2}$, Rúbio Sabino Bruzzi ${ }^{1}$, André Gustavo Pereira \\ de Andrade ${ }^{2}$
}

\section{João Gabriel da Silveira- Rodrigues ${ }^{1, *}$, Camila Cristina Melo ${ }^{2}$, Rúbio Sabino Bruzzi ${ }^{1}$, André Gustavo Pereira de Andrade ${ }^{2}$}

\begin{abstract}
'Exercise Physiology Laboratory, Federal University of Minas Gerais, School of Physical Education, Physiotherapy and Occupational Therapy, Av. Pres, Antônio Carlos, 6627 - São Luiz, Belo HorizonteMG State, BRAZIL.

'Laboratory of Biomechanics Federal University of Minas Gerais, School of Physical Education, Physiotherapy and Occupational Therapy, Av. Pres. Antônio Carlos, São Luiz, Belo Horizonte-MG State, BRAZIL.
\end{abstract}

\section{*Correspondence}

MSc. João Gabriel da SilveiraRodrigues

Exercise Physiology Laboratory. Federal University of Minas Gerais. School of Physical Education, Physiotherapy and Occupational Therapy. Av. Pres. Antônio Carlos, 6627 - São Luiz, Belo Horizonte - MG State, BRAZIL.

Phone: +55 31983911654

Email: joaogabrielsrod@gmail.com

\section{History}

- Submission Date: 12-04-2020:

- Review completed: 24-05-2020;

- Accepted Date: 19-06-2020.

\section{DOI : 10.5530/ijcep.2020.7.2.17}

Article Available online http://www.ijcep.org

\section{Copyright}

(c) 2020 Phcog.Net. This is an openaccess article distributed under the terms of the Creative Commons Attribution 4.0 International license.

\begin{abstract}
Background and Aim: In everyday practice, the anthropometric assessment including measures of skinfolds and body girths must be performed avoiding previous physical exercise. However, few studies are conducted using situations with ecological validity. Thereby, this study aimed to compare if $10 \mathrm{k}$ treadmill run alters skinfold measures and body girths of runners. Methods: Eighteen runners of both sexes and levels $(34 \pm 6$ years old with $8 \pm 7$ years of run practice and $63.6 \pm 8.8 \mathrm{ml}^{*} \mathrm{~kg}-1^{*} \mathrm{~min}-1$ of $\mathrm{VO}_{2} \mathrm{max}$ ) underwent body girths, skinfolds thickness and body weight assessments before and after the 10k run. The anthropometric parameters were compared by Student's t-Test dependent for paired samples and correlations between body weight reduction and anthropometric parameters were compared by Pearson's correlation coefficient with $a=5 \%$. Results: The 10k run reduces $1.4 \%$ of body weight. However, no significant changes were observed in the sum of skinfold thickness or body girths. The percentage of body weight reduction also was not correlated with the exercise-induced changes in skinfold thickness and body girths. Conclusion: Although 10k run causes a body weight reduction, the skinfold thickness and body girths not altered in previously trained individuals and was not correlated with body weight reductions. In this way, the use of these anthropometric assessment techniques can occur both before and after a conventional training session in runners.
\end{abstract}

Key words: Anthropometric assessment, Body girths, Body fat, Prolonged exercise, Body composition.

\section{INTRODUCTION}

In sports and fitness contexts, anthropometry assessment is widely used to monitoring the general health of athletes ${ }^{[1]}$ in the selection phase, preseason and follow-up. ${ }^{[2]}$ Among the procedures that commonly used for the anthropometric assessment, the measurement of skin folds and body girths, a lowcost technique, that when performed under accuracy methodological rigor shows good reliability for assessing body composition. ${ }^{[3]}$ Through equations that consider the skinfold thickness, the body density is initially inferred ${ }^{[4,5]}$ and then both body fat percentage and muscle mass are obtained. ${ }^{[6]}$

Regarding the guidelines given to ensure an adequate reproducibility of anthropometric assessments, it is widely suggested that any type of physical exercise should be avoided in the hours prior to the assessment. ${ }^{[3,7-9]}$ It may be justified by the accumulation of extracellular water, a result of peripheral vasodilation due to aerobic exercise, which could increase the skinfold thickness. ${ }^{[9]}$

Previous studies reinforce the hypothesis of the reduction of skinfolds after an exercise practice, ${ }^{[10-12]}$ particularly when subjected the volunteers to extreme

situations, such long duration competitions, lasting many hours to more than one day. Despite this, the mechanisms that explain the exercise-induced changes in anthropometric measures are poorly known. ${ }^{[13]}$ To the best of our acknowledgment, none research on the evaluation of skinfolds thickness and body circumferences after a single short-duration exercise session with wide reproducibility was found in the literature. Thus, studies that promote greater understanding and support the applicability of this technique in different contexts, such as after a conventional aerobic training session, can contribute and amplify its use in the most varied contexts. Thereby, the present study aimed to compare skinfold thickness and body girths before and after a 10k treadmill run.

\section{MATERIALS AND METHODS}

\section{Sample Characteristics and Ethical Considerations}

Eighteen elite and recreational brazilian runners (2345 years old) of both sexes, participated in the study

Cite this article: Silveira-Rodrigues JG, Melo CC, Bruzzi RS, Andrade AGP. A Single 10k Run Session does not Change either Skinfold Thickness or Body Circumferences. Int J Clin Exp Physiol. 2020;7(2):69-72. 
(Table 1). The volunteers signed a free and informed consent form where the research procedures were exposed, as well as their risks and benefits, which were approved by the Research Ethics Committee of the Federal University of Minas Gerais under no. 64800817.0.0000.5149. All the subjects who completed the $10 \mathrm{k}$ run on a treadmill were included in the analysis. The procedures were performed in the Laboratory of Exercise Physiology of Federal University of Minas Gerais.

\section{Procedures}

The experimental procedures were conducted in two different days and the subjects abstained from physical exercises in the $24 \mathrm{hr}$ before the experimental procedures. At first day, a progressive test was performed to determine the maximum oxygen consumption $\left(\mathrm{VO}_{2} \max \right)^{[14]}$ and maximal heart rate. On the second day, the $10 \mathrm{k}$ run on a treadmill was performed and the skinfold thickness, body circumferences, body weight and height were assessed. Subsequently, the subjects performed a $10 \mathrm{k}$ treadmill run (93T Life Fitness, Illinois, USA) with a $1 \%$ inclination in a controlled ambient (18-20 Celsius degree and 65-75\% of humidity). Each subject should run the $10 \mathrm{k}$ as fast as possible in self-paced velocity without ingestion of water. Heart rate (HR) was recorded every minute for the calculation of mean HR and the highest HR value was considered as maximal HR. Immediately after the exercise, the same evaluator conducted the sequence of procedures identical to those performed at the pre-exercise moment.

\section{Anthropometric Assessment}

For the skinfold thickness assessment, a pen mark was made on the skin at predetermined locations, which remained after the $10 \mathrm{~km}$ run. The skinfolds were evaluated with dry skin on the right side by the skinfold (Lange $^{\circledast}$ ) graduated from $0-65 \mathrm{~mm}$ and $1 \mathrm{~mm}$ accuracy was measured for the measurements. These procedures were carried out following the proposition of Norton and Olds. ${ }^{[3]}$ Three measurements were obtained for each anatomical point and the mean value of these measurements was considered for statistical analysis. The caliper was positioned for reading about $1 \mathrm{~cm}$ below the fingers that pinched the fold, composed only of skin and fat. Two seconds after its placement, the reading was performed on the nearest millimeter. Nine skinfolds sites were assessed in the present study (subscapular, tricipital, bicipital, pectoral, axillary, supra-iliac, abdominal, thigh, calf), according to Jackson and Pollock, ${ }^{[5]}$ the most used protocols for estimation of body density in practical and research contexts in sports and fitness. Then, the Siri equation ${ }^{[6]}$ was used to calculate the body fat percent.

Body circumferences were evaluated using a $1.5 \mathrm{~m}$ tape and $0.5 \mathrm{~cm}$ of accuracy was considered in the evaluations. The volunteers remained standing, adopting a relaxed posture and under involuntary control of their breathing. The sites evaluated were the shoulder, arm, abdomen, hip, thigh and calf, following international standards. ${ }^{[3,8]}$

The body weight was evaluated with the volunteer barefoot and wearing only underwear with a precision of $20 \mathrm{~g}$ (digital scale, MF100, Filizola ${ }^{\oplus}$ ). The height was evaluated by the stadiometer (Line 31, Filizola ${ }^{\circ}$ ) adopting precision of $1 \mathrm{~cm}$. At the end of the exercise, the volunteers were instructed to dry themselves with paper towels before the assessment of body weight, circumferences and skinfolds.

\section{Statistical Analysis of Data}

The normality and homoscedasticity were met, which made it possible to perform parametric analysis. All data are expressed as mean \pm standard deviation. Additionally, to compare the values of each of the skinfolds and the circumferences as well as their sum before and after the $10 \mathrm{~km}$ race in each of the groups, the paired Student's t-test with two-tailed was used in a significance level of 5\%. The intra-class correlation coefficient analysis was used to evaluate the reproducibility between the three measurements performed in each of the skinfolds at both the pre- and post-exercise moments. For ICC analysis, the mixed model was admitted, assuming that the skinfolds and circumferences were measured by the same evaluator in the pre- and post-exercise moments and the variation would occur only in the measurements. Pearson's correlation was used to measure the relationship between changes in body weight caused by exercise and changes in skinfolds. The inferential analysis and graphs were performed in Sigma Plot 13.0 software.

\section{RESULTS}

There was no significant changes in any of the skinfolds (Table 2) or body circumferences (Table 3 ) as well as in their sums were observed ( $p>0.05$ for both). Men showed the lower sum of skinfold thickness (Table 2) and the higher sum of body circumferences (Table 3$)$ than women $(p<0.05$ for both). Elite athletes also showed a lower sum of skinfold thickness (Table 2) and the higher sum of body circumferences (Table 3) than women ( $p<0.02$, for both). Additional analyses were conducted and none of the subgroups showed any changes in the skinfold thickness or body circumferences ( $p>0.05$ for all).

The ICC analysis showed significant reliability among the three measures in all the nine anatomical locations used for skinfold measures both pre ( 0.96 to 0.99 for each of the measures) and post-exercise times (0.95 to 0.98 for each of the measures).

Characteristics of the $10 \mathrm{k}$ treadmill run were described in Table 4 . The means comparison was performed only for the exercise-induced body weight changes and showed that all groups reduced your body weight after exercise $(p<0.05)$. Although the exercise reduces body weight, the

Table 1: Characteristics of the subjects (mean \pm standard deviation).

$\begin{array}{cccccc} & \begin{array}{c}\text { Men } \\ (n=9)\end{array} & \begin{array}{c}\text { Women } \\ (n=9)\end{array} & \begin{array}{c}\text { Elite } \\ (n=6)\end{array} & \begin{array}{c}\text { Recreational } \\ (n=12)\end{array} & \begin{array}{c}\text { All } \\ (n=18)\end{array} \\ \text { Age (years) } & 36.4 \pm 3.5 & 31.7 \pm 7.3 & 31.7 \pm 6.4 & 35.3 \pm 5.8 & 34.1 \pm 6.1 \\ \text { Weight }(\mathrm{kg}) & 71.8 \pm 8.4 & 57.1 \pm 7.4 & 58.9 \pm 7.7 & 67.2 \pm 11.3 & 64.5 \pm 10.8 \\ \begin{array}{c}\text { Height }(\mathrm{cm}) \\ \text { Body fat }(\%)\end{array} & 175 \pm 7.7 & 161.4 \pm 7.5 & 168 \pm 11.3 & 168.3 \pm 10.0 & 168.2 \pm 10.1 \\ \mathrm{VO}_{2} \max \left(\mathrm{ml}^{*} \mathrm{~kg}\right. & 69.5 \pm 7.5 & 57.7 \pm 5.4 & 70.1 \pm 7.2 & 60.3 \pm 7.7 & 63.6 \pm 8.8 \\ \begin{array}{c}\left.{ }^{*} \min ^{-1}\right) \\ \mathrm{HRmax}(\mathrm{bpm})\end{array} & 184 \pm 9 & 179 \pm 7 & 175 \pm 6 & 184 \pm 7 & 181 \pm 8 \\ \begin{array}{c}\text { Time of practice } \\ \text { (years) }\end{array} & 10.6 \pm 8.2 & 6.0 \pm 4.2 & 11.6 \pm 9.6 & 6.7 \pm 4.4 & 8.3 \pm 6.7\end{array}$

Note: HR: Heart rate, BPM: Beats per minute 
Table 2: Skinfold thickness $(\mathrm{mm})$ before and after exercise (mean \pm standard deviation).

\begin{tabular}{|c|c|c|c|c|c|c|c|c|}
\hline & \multicolumn{2}{|c|}{$\begin{array}{l}\text { Men } \\
(n=9)\end{array}$} & \multicolumn{2}{|c|}{$\begin{array}{c}\text { Women } \\
(n=9)\end{array}$} & \multicolumn{2}{|c|}{$\begin{array}{l}\text { Elite } \\
(n=6)\end{array}$} & \multicolumn{2}{|c|}{$\begin{array}{l}\text { Recreational } \\
\qquad(n=12)\end{array}$} \\
\hline & Pre & Post & Pre & Post & Pre & Post & Pre & Post \\
\hline Subescapular & $13 \pm 7$ & $13 \pm 7$ & $11 \pm 3$ & $10 \pm 5$ & $10 \pm 4$ & $11 \pm 7$ & $14 \pm 6$ & $14 \pm 6$ \\
\hline Tricipital & $8 \pm 4$ & $8 \pm 4$ & $17 \pm 3$ & $16 \pm 4$ & $12 \pm 6$ & $11 \pm 6$ & $14 \pm 5$ & $14 \pm 5$ \\
\hline Pectoral & $6 \pm 3$ & $6 \pm 3$ & $7 \pm 3$ & $6 \pm 2$ & $5 \pm 2$ & $5 \pm 2$ & $8 \pm 2$ & $7 \pm 2$ \\
\hline Bicipital & $4 \pm 1$ & $4 \pm 1$ & $7 \pm 2$ & $7 \pm 2$ & $5 \pm 2$ & $6 \pm 2$ & $6 \pm 2$ & $6 \pm 2$ \\
\hline Axillary & $8 \pm 4$ & $8 \pm 5$ & $7 \pm 2$ & $7 \pm 2$ & $7 \pm 3$ & $6 \pm 3$ & $9 \pm 4$ & $9 \pm 3$ \\
\hline Suprailiac & $11 \pm 6$ & $11 \pm 6$ & $10 \pm 3$ & $10 \pm 2$ & $8 \pm 4$ & $8 \pm 4$ & $12 \pm 5$ & $13 \pm 4$ \\
\hline Abdominal & $16 \pm 10$ & $16 \pm 9$ & $15 \pm 5$ & $15 \pm 5$ & $10 \pm 6$ & $10 \pm 6$ & $19 \pm 6$ & $19 \pm 6$ \\
\hline Thigh & $12 \pm 7$ & $12 \pm 8$ & $21 \pm 5$ & $22 \pm 7$ & $15 \pm 9$ & $14 \pm 9$ & $19 \pm 7$ & $20 \pm 8$ \\
\hline Calf & $8 \pm 6$ & $8 \pm 5$ & $9 \pm 5$ & $12 \pm 5$ & $10 \pm 6$ & $10 \pm 6$ & $12 \pm 5$ & $11 \pm 5$ \\
\hline Sum & $86 \pm 43$ & $86 \pm 44$ & $108 \pm 22$ & $107 \pm 22$ & $81 \pm 40$ & $79 \pm 41$ & $113 \pm 28$ & $112 \pm 29$ \\
\hline
\end{tabular}

Table 3: Body girths $(\mathrm{cm})$ before and after exercise (mean \pm standard deviation).

\begin{tabular}{|c|c|c|c|c|c|c|c|c|}
\hline & \multicolumn{2}{|c|}{$\begin{array}{l}\text { Men } \\
(n=9)\end{array}$} & \multicolumn{2}{|c|}{$\begin{array}{c}\text { Women } \\
(n=9)\end{array}$} & \multicolumn{2}{|c|}{$\begin{array}{l}\text { Elite } \\
(n=6)\end{array}$} & \multicolumn{2}{|c|}{$\begin{array}{l}\text { Recreational } \\
\qquad(n=12)\end{array}$} \\
\hline & Pre & Post & Pre & Post & Pre & Post & Pre & Post \\
\hline Shoulders & $101 \pm 12$ & $100 \pm 12$ & $87 \pm 7$ & $87 \pm 7$ & $92 \pm 14$ & $92 \pm 13$ & $94 \pm 12$ & $94 \pm 11$ \\
\hline Arms $R$ & $31 \pm 4$ & $30 \pm 3$ & $27 \pm 2$ & $27 \pm 1$ & $27 \pm 2$ & $27 \pm 1$ & $30 \pm 3$ & $30 \pm 3$ \\
\hline Arms $L$ & $30 \pm 3$ & $30 \pm 3$ & $27 \pm 2$ & $27 \pm 2$ & $27 \pm 2$ & $27 \pm 2$ & $29 \pm 3$ & $29 \pm 3$ \\
\hline Abdominal & $81 \pm 7$ & $80 \pm 7$ & $75 \pm 5$ & $74 \pm 6$ & $73 \pm 2$ & $72 \pm 2$ & $80 \pm 7$ & $80 \pm 7$ \\
\hline Hip & $95 \pm 4$ & $95 \pm 3$ & $93 \pm 5$ & $93 \pm 5$ & $90 \pm 3$ & $91 \pm 4$ & $96 \pm 4$ & $96 \pm 4$ \\
\hline Thigh $R$ & $54 \pm 4$ & $54 \pm 4$ & $51 \pm 4$ & $51 \pm 4$ & $50 \pm 2$ & $50 \pm 2$ & $54 \pm 4$ & $54 \pm 4$ \\
\hline Thigh L & $54 \pm 4$ & $53 \pm 3$ & $51 \pm 3$ & $51 \pm 4$ & $50 \pm 2$ & $50 \pm 2$ & $54 \pm 4$ & $54 \pm 4$ \\
\hline Calf $R$ & $37 \pm 2$ & $37 \pm 2$ & $35 \pm 3$ & $35 \pm 2$ & $35 \pm 3$ & $35 \pm 2$ & $35 \pm 2$ & $36 \pm 2$ \\
\hline Calf $L$ & $37 \pm 2$ & $37 \pm 2$ & $35 \pm 2$ & $35 \pm 2$ & $35 \pm 2$ & $35 \pm 2$ & $35 \pm 2$ & $36 \pm 2$ \\
\hline Sum & $526 \pm 24$ & $521 \pm 25$ & $479 \pm 27$ & $479 \pm 27$ & $479 \pm 27$ & $477 \pm 26$ & $511 \pm 32$ & $508 \pm 32$ \\
\hline
\end{tabular}

Note: R: Right side, L: Left side.

Table 4: Characteristics of the exercise protocol (mean \pm standard deviation).

\begin{tabular}{cccccc} 
& $\begin{array}{c}\text { Men } \\
(n=9)\end{array}$ & $\begin{array}{c}\text { Women } \\
(n=9)\end{array}$ & $\begin{array}{c}\text { Elite } \\
(n=6)\end{array}$ & $\begin{array}{c}\text { Recreational } \\
(n=12)\end{array}$ & $\begin{array}{c}\text { All } \\
(n=18)\end{array}$ \\
\hline 10k run (mins) & $45.3 \pm 5.3$ & $48.9 \pm 4.8$ & $42 \pm 3.7$ & $49 \pm 3.9$ & $47 \pm 5.1$ \\
Hr mean (bpm) & $160 \pm 15$ & $168 \pm 12$ & $154 \pm 16$ & $169 \pm 10$ & $164 \pm 14$ \\
HR (\% max) & $89.3 \pm 4.8$ & $94.0 \pm 3.9$ & $91.5 \pm 5.3$ & $92.1 \pm 4.8$ & $91.9 \pm 4.8$ \\
$\Delta$ BW (\%) & $-1.4 \pm 0.3^{*}$ & $-1.3 \pm 0.5^{*}$ & $-1.6 \pm 0.4^{*}$ & $-1.3 \pm 0.4^{*}$ & $-1.4 \pm 0.4^{*}$ \\
\hline
\end{tabular}

Note: HR: Heart rate, BW: Body weight.

${ }^{*}$ Student's $t$ test was significant different $(p<0.05)$ in pre $v$ s. post

relationship between the change in exercise-induced body weight and the change in the sum of all skinfolds $(r=0.05 ; p=0.82)$ and the sum of all body circumferences $(r=-0.31 ; p=0.22)$ were not significant.

\section{DISCUSSION}

The present study was investigated whether a single session of running alters the skinfold thickness or body girths of runners. In contrast to previous data ${ }^{[10-12]}$ although $10 \mathrm{k}$ treadmill run reduces $1.4 \%$ of body weight there were not found any differences in the skinfold thickness or body girths even when subjects were split into subgroups. Also, exercise-induced body weight changes were not related to changes in anthropometric measures.

Skinfold thickness is an indirect method of assessing body composition and the percentage of body fat. Studies confirming the validity of skinfolds based on their relationship to hydrostatic weighting, is considered to be a gold standard method. ${ }^{[13]}$ This method is based on the relationship between subcutaneous fat, internal fat and body density. It is possible to predict the amount of body fat through regression equations ${ }^{[9]}$ that consider several measures of skinfold thickness. However, it is necessary to test the intra-rater reliability of this measures ${ }^{[13]}$ and in the 
present study, a good intra-class correlation coefficient (0.96 to 0.99) was obtained for all skinfolds and body circumference evaluations, confirming the good reliability of the technique used.

In the present study, despite the reduction of body weight caused by exercise, there was no change in the cutaneous skinfolds and eight body circumferences isolated and also as a sum after the accomplishment of $10 \mathrm{k}$ in the treadmill. General guidelines suggest that anthropometric assessment should be performed avoiding previous exercise, ${ }^{[7-9]}$ but conflicting results are reported in the literature ${ }^{[10-12,15]}$ which, in turn, many studies are conducted with strenuous exercise duration ${ }^{[10-12]}$ with little ecological validity. Knechtle et al. ${ }^{[10]}$ carried out a study with 148 athletes who completed the $100 \mathrm{k}$ ultra marathon evaluating them before and after completing this distance. Similar to our findings, the authors did not find reduction in the sum of eight skinfolds, although there were observed isolated reductions in the circumference and skinfold thickness in both upper and lower body. In contrast to these results, the other two studies showed a decrease in skin folds after long-term competitive exercise protocols, such as ultra-distance cycling ${ }^{[11]}$ and in the open water swimming events. ${ }^{[12]}$

A possible explanation for this controversial result is the great disparity between the duration of the activities, being of $47 \mathrm{~min}$ in the present study and approximately $12 \mathrm{hr}$ in ultra-marathon, ${ }^{[10]}$ about $26 \mathrm{hr}$ in the ultra-distance cycling ${ }^{[11]}$ and $9 \mathrm{hr}$ in the swimming in open waters. ${ }^{[12]} \mathrm{It}$ is reported that a longer duration of the test would be inversely associated with alterations in abdominal skinfold. ${ }^{[1]}$ Thus, the duration of activity seems to be relevant to promote a reduction of body circumferences and skinfolds and $10 \mathrm{~km}$ run it does not seem to be enough to alter anthropometric measurements.

Contradictory results were observed between the present study and the previous literature. It may also have occurred due to the lower reduction of body weight, being $1.4 \%$ in the present study versus $1.7 \%$ found in Bischof et al..$^{[1]}$ and $2.5 \%$ found by Knechtle et al. ${ }^{[10]}$ The sweat production is mediated by the autonomic nervous system to counteract the increase in internal temperature promoted by muscle contractions, inducing losses of water content in their bodies.

Each kilogram lost immediately after exercise compared to pre-exercise ranges between 1 and $2 \%^{[16]}$ the body's hydration status, explaining the reduction in body weight loss after exercise. Body weight reductions were directly related to the reduction in pectoral, triceps and thigh skinfold thickness as well as a reduction in the circumference of the arms, thigh and legs. ${ }^{[10]}$ Also, these changes may be due to a reduction in the specific density of urine, a marker of body dehydration, which is related to the reduction of the axillary skinfold. ${ }^{[10]}$ In the present study, there was no reduction in skinfolds and no correlation between the changes in body weight and changes in the thickness of skinfolds or circumferences after $10 \mathrm{k}$ of running, it may be that the proposed activity, chosen because of its greater ecological validity seems not to have been sufficient to promote body dehydration at levels similar to those observed by Knechtle et al. ${ }^{[10]}$ explaining the absence of changes in the anthropometric measures.

In the present study, the sample population had low amounts of body fat, as demonstrated in runner-athletes, reported by Garrido-Chamorro et al..$^{[2]}$ Despite this data, the additional analysis revealed that both men $v$ s. women and recreational vs. elite runners who had different body compositions in baseline seem not to have their anthropometric measures influenced by the previous $10 \mathrm{k}$ run on a treadmill.

Running involves the displacement of the own body weight, the skinfold assessment is an important part of the process of training monitoring of athletes because it allows adequate controlling of their body composition.
In this way, the present study suggests that an anthropometric assessment can be performed before and after a conventional training running session. These findings are encouraged to be replicated in future investigations that use other exercise models (e.g. strength training sessions) and other populations (e.g. as sedentary or recreational athletes of other disciplines) to confirm the influence of a single exercise session on skinfold thickness and body circumferences.

\section{Limitations of the Study}

Although the water ingestion was not allowed during the 10k run, the lack of estimation of the hydration status and time spent between the last meals, are the limitations of the study.

\section{CONCLUSION}

Skinfolds and body circumferences do not change with prior aerobic exercise in athletes or recreational subjects, suggesting that the use of these techniques for anthropometric assessment can occur both before and after a training session.

\section{ACKNOWLEDGEMENT}

For the members of Exercise Physiology members and Laboratory of Biomechanics for the contribution in data collection.

\section{CONFLICT OF INTEREST}

The authors declare that they have no conflict of interest.

\section{ABBREVIATIONS}

$\mathrm{VO}_{2 \max }$ : Maximum oxygen consumption; HR: Heart rate.

\section{REFERENCES}

1. Giampietro M, Ebner E, Bertini I. The clinical significance of body composition and anthropometric evaluation in athletes. Med J Nutrition Metab. 2011:4(2):93-

2. Garrido-Chamorro R, Sirvent-Belando JE, González-Lorenzo M, Blasco-Lafarga C, Roche E. Skinfold sum: Reference values for top athletes. Int J Morphol. 2012;30(3):803-9.

3. Norton K, Olds T. Antropometrica. Porto Alegre: Artmed. 2005.

4. Guedes DP. Study of Body Fat by Measuring the Values of Body Density and Skinfold Thickness and University Students. Kinesis. 1985;1:183-212.

5. Jackson AS, Pollock ML. Generalized equations for predicting body density of men. Br J Nutr. 1978;40(3):497-504

6. Siri WE. Body volume measurement by gas dilution. Techniques for measuring body composition. National Academy of Sciences. 1961;11:108-17.

7. Keys A, Brozek J. Body fat in adult man. Physiol Rev. 1953;33(3):245-325

8. Heyward VH, Stolarczyk LM. Assessment of Applied Body composition. Champaign: Human Kinetics. 1996.

9. Eduardo C, Machado P, Caputo F, DeLucas RD, Denadai BS. Physiological and anthropometric factors associated with uphill performance in off road cycling. $R$ Bras Cien Mov. 2002;10:35-50.

10. Knechtle B, Baumgartner $S$, Knechtle $P$, Rüst CA, Rosemann T, Bescós R. Changes in single skinfold thickness in $100 \mathrm{~km}$ ultramarathoners. Open Access J Sports Med. 2012;3:147-57.

11. Bischof M, Knechtle $B$, Rüst CA, Knechtle $P$, Rosemann T. Changes in skinfold thicknesses and body fat in ultra-endurance cyclists. Asian J Sports Med. 2013;4(1):15-22.

12. Weitkunat T, Knechtle B, Knechtle P, Rüst CA, Rosemann T. Body composition and hydration status changes in male and female open-water swimmers during an ultra-endurance event. J Sports Sci. 2012;30(10):1003-13.

13. Sichieri R, Fonseca VDM, Lopes CS. How to measure the reliability of skinfolds Rev Bras Epidemiol. 1999:2:82-9.

14. Svedahl K, Maclntosh BR. Anaerobic threshold: The concept and methods of measurement. Can J Appl Physiol. 2003;28(2):299-323.

15. Harrop BJ, Woodruff SJ. Effects of acute and 2-hr post-physical activity on the estimation of body fat made by the bod pod. J Strength Cond Res. 2015:29(6):1527-33

16. Coyle EF. Substrate utilization during exercise in active people. The Am J Clin Nutr. 1995;61(4):968S-79S. 